\title{
Estabilidade Temporal dos Escores de Interesses Profissionais: Estudo Longitudinal
}

\author{
Karen Cristina Alves Lamas ${ }^{1}$ \\ Universidade Salgado de Oliveira, Juiz de Fora-MG, Brasil \\ Ana Paula Porto Noronha \\ Universidade São Francisco, Campinas-SP, Brasil
}

\section{RESUMO}

Estudos longitudinais para investigação dos interesses profissionais não são frequentes no contexto nacional. O presente estudo teve como objetivo analisar a estabilidade temporal das dimensões da Escala de Aconselhamento Profissional - EAP e dos escores de nitidez e elevação. Foram utilizados os dados de 96 estudantes, obtidos por meio de três aplicações, uma a cada ano do ensino médio. Verificaram-se coeficientes moderados de estabilidade para as dimensões e para os índices de nitidez, e fracos para elevação. Uma análise de variância evidenciou a estabilidade dos escores, com leve aumento dos níveis de nitidez. Ressalta-se que, ao analisar os resultados, é necessário considerar aspectos teóricos relacionados aos interesses profissionais e a estratégia de construção do instrumento.

Palavras-chave: psicometria; estudantes de ensino médio; desenvolvimento profissional; orientação vocacional; interesses profissionais.

\section{ABSTRACT - Temporal Stability of Scores of Vocational Interests: Longitudinal Study}

Longitudinal studies for the investigation of professional interests are not frequent in the national context. The present study aimed to analyze the temporal stability of the dimensions of the Professional Counseling Scale (PCS), and the differentiation and elevation scores. The data of 96 students were obtained through three applications, each representing a different year of high school. In the dimensions and acuity indexes, moderate stability coefficients, and weak for elevation. Variance analysis showed score stability, with a slight increase in acuity levels. It should be emphasized that when analyzing the results it is necessary to consider theoretical aspects related to professional interests and the instrument's construction strategy.

Keywords: psychometry; high school students; professional development; vocational guidance; professional interests.

\section{RESUMEN - Estabilidad Temporal de Puntajes de Interés Profesional: Estudio Longitudinal}

Los estudios longitudinales para la investigación de intereses profesionales no son frecuentes en el contexto nacional. El presente estudio tuvo como objetivo analizar la estabilidad temporal de las dimensiones de la Escala de Asesoramiento Profesional - EAP y de los puntajes de nitidez y elevación. Se utilizaron los datos de 96 estudiantes, obtenidos por medio de tres aplicaciones, realizadas, cada año de Enseñanza Secundaria. Se verificaron coeficientes moderados de estabilidad para las dimensiones y para los índices de nitidez, y coeficientes más débiles para elevación. Un análisis de varianza mostró la estabilidad de los puntajes, con leve aumento de los niveles de nitidez. Se señala que al analizar los resultados, es necesario considerar aspectos teóricos relacionados a los intereses profesionales y a la estrategia de construcción del instrumento.

Palabra clave: psicometría; estudiantes de Enseñanza Secundaria; desarrollo profesional; orientación vocacional; intereses profesionales.

Os inventários que avaliam interesses profissionais são instrumentos de grande utilidade para a prática do psicólogo, sendo utilizados em processos de seleção de pessoas, de recolocação profissional, de preparação para a aposentadoria e, principalmente, em orientação profissional e de carreira. Nesse último contexto, a avaliação dos interesses pode ser realizada com diferentes finalidades, tais como proporcionar autoconhecimento, analisar a qualidade e quantidade de informações ocupacionais do orientando, identificar novos interesses ou estreitar opções profissionais, bem como confirmar escolhas realizadas (Hansen, 2005).

Para que o resultado dessas avaliações seja confiável, os instrumentos utilizados devem apresentar qualidades psicométricas que garantam sua cientificidade (Noronha, Freitas, \& Ottati, 2003). A Escala de Aconselhamento Profissional - EAP é um teste que afere os interesses profissionais e objeto de investigação do presente estudo. Embora seja um instrumento que passou por muitas pesquisas (Noronha \& Nunes, 2012; Noronha, Ottati,

Endereço para correspondência: Universidade Salgado de Oliveira - UNIVERSO. Avenida dos Andradas, 731, Morro da Glória, 36036-000, Juiz de Fora-MG. Tel.: (32) 3311-9917. E-mail: karen_lammas@yahoo.com.br

Trabalho derivado da tese de doutorado da autora, desenvolvida com apoio da Fundação de Amparo à Pesquisa do Estado de São Paulo - FAPESP e da Coordenação de Aperfeiçoamento de Pessoal de Nível Superior - CAPES. 
Mansão, \& Cezar, 2011; Noronha, Pinto, \& Luca, 2015; Noronha, Sisto, \& Santos, 2007, 2012; Sartori, Noronha, Godoy, \& Ambiel, 2010; Sisto, Santos, \& Noronha, 2009), a EAP ainda não foi submetida a um estudo longitudinal que permita analisar a precisão de seus escores a partir de características diretamente associadas ao construto que pretende medir, como exemplo, a estabilidade. Pesquisas que confirmem a estabilidade dos interesses profissionais combinados com a investigação da confiabilidade do instrumento fornecem aos profissionais mais segurança nos resultados da avaliação, especialmente quando é intenção predizer escolhas (Hansen, 2005).

De acordo com Savickas (1999), os interesses profissionais podem ser entendidos como um traço e como um estado. Enquanto estado de consciência, o construto é caracterizado por uma prontidão para pensar ou responder a um estímulo específico do ambiente, como objetos, atividades, pessoas e experiências. Após realizar avaliações afetivas e cognitivas, o indivíduo pode ter expectativas de que o contato com o referido estímulo irá trazer satisfação pessoal e incorporá-lo ao seu sistema de autoconceitos, junto a outros estímulos semelhantes. Ocorrerá, portanto, uma disposição para responder a um grupo homogêneo de estímulos ambientais, de modo consistente e estável, caracterizando-o como traço.

Segundo o autor, por meio de inventários de interesses, é possível definir a força relativa, ou seja, o quanto uma preferência por determinada atividade se sobressai quando comparada a outros interesses. Ter consciência dessa disposição conduz à localização de um código no mundo do trabalho e ao desenvolvimento do sistema de autoconceitos existente. A consciência dessa representação promove a estabilidade e a continuidade da disposição (Savickas, 1999).

Os itens da EAP foram construídos tendo por base o interesse como estado (Noronha et al., 2007). São apresentados vários estímulos na forma de atividades profissionais, para os quais o respondente deve fazer uma avaliação do quanto ele gostaria de desenvolvê-las, ou seja, entrar em contato com cada uma das atividades. Essas atividades são agrupadas de forma que se relacionam a determinadas áreas profissionais, assim, o nível de preferência em cada uma das áreas denota os interesses profissionais do indivíduo. Esse tipo de inventário também é chamado de escala de interesses básicos (Rounds \& Su, 2014).

Ressalta-se que os interesses profissionais se tornam estáveis ao final da adolescência ou no início da idade adulta (Super, 1957) e, em alguns casos específicos, ainda podem ser modificados após essa fase (Lent, Brown, \& Hackett, 1994). No contexto internacional, pesquisas sobre a estabilidade dos interesses profissionais são publicadas, pelo menos, desde a década de 1970 (Wang \& Wanberg, 2017). O estudo de metanálise realizado por Low, Yoon, Roberts e Rounds (2005) mostrou que até o final da adolescência os índices de estabilidade estão entre 0,50 e 0,60 e, após os 18 anos, os coeficientes aumentam para 0,70 . Ainda que haja flutuações, pressupõe-se que o construto adquira certa estabilidade ao longo tempo (Lent et al., 1994; Rounds \& Su, 2014) e isso deve ser refletido por sua medida. Assim, a fidedignidade teste-reteste é um parâmetro importante para os instrumentos que avaliam interesses (Urbina, 2007).

De acordo com Low e Rounds (2007), há cinco formas de avaliar a estabilidade dos interesses profissionais, por meio da análise rank-order, de nível, de perfil, estrutural e de congruência. Destacam-se aqui as duas primeiras por estarem relacionadas aos objetivos deste estudo. A estabilidade rank-order refere-se à correlação dos escores em cada dimensão de interesses obtidos em dois momentos diferentes (teste-reteste), os índices representam as mudanças de posicionamento relativo dos indivíduos dentro do grupo. Já a estabilidade de nível consiste em analisar se os escores dos indivíduos aumentaram ou diminuíram em diferentes domínios de interesses ao longo do tempo.

Outra característica dos interesses que pode auxiliar na avaliação da estabilidade são os pressupostos ou conceitos secundários derivados da teoria de Holland (1997). Os conceitos secundários desenvolvidos por Holland (1997) são denominados como nitidez/diferenciação, consistência, congruência e identidade. A elevação do perfil de interesses (Fuller, Holland, \& Johnston, 1999) tem sido discutida como outro conceito secundário importante (Bullock \& Reardon, 2005; Jaensch, Hirschi, \& Spurk, 2016). No presente trabalho, foi possível e adequado aplicar aos escores da EAP os índices que indicam a nitidez dos interesses e a elevação. O primeiro se refere à clareza do perfil de interesses, ou seja, dentre as dimensões ou tipos avaliados por um teste, verifica-se a distância entre o maior e o menor escore; quanto maior esse valor, ou seja, a nitidez, maior a capacidade preditiva dos interesses para com a escolha da profissão (Holland, 1997). O segundo diz respeito ao nível geral de interesses de uma pessoa em diferentes campos, ou seja, a soma dos escores obtidos em cada dimensão analisada (Fuller et al., 1999). A elevação do perfil está associada a diversos fatores, como personalidade (p.e., impulsividade e desejo de se expressar), sucesso acadêmico e energia que o indivíduo investe no processo de tomada de decisão profissional (Bullock \& Reardon, 2005), flexibilidade dos interesses (Tracey, 2012), autoeficácia ocupacional e engajamento na carreira (Jaensch et al., 2016), oferecendo informações adicionais a serem utilizadas nos processos de orientação.

No Brasil, há poucos estudos sobre a estabilidade do perfil de interesses profissionais e os construtos secundários de Holland (1997), apenas dois foram recuperados. Mansão e Yoshida (2006) investigaram a estabilidade temporal da versão brasileira do Self-Directed Search Career Explorer - SDS (Questionário de Busca Autodirigida) em uma amostra composta por 122 estudantes do ensino médio. Com reaplicação do instrumento em sete 
dias, a variação dos índices de correlação para cada um dos seis tipos (dimensões de interesses) que compõem o teste foi de 0,82 a 0,91. Noronha e Ambiel (2015), por sua vez, investigaram a nitidez/diferenciação do perfil de interesses por meio de um banco de dados com 6824 pessoas que responderam a EAP. Eles verificaram que o índice de nitidez foi maior entre universitários, seguidos por estudantes de ensino fundamental e estudantes de ensino médio e, mais baixos em pessoas que já haviam completado essa etapa de ensino e não ingressaram no ensino superior.

Algumas pesquisas realizadas no contexto internacional empregaram delineamentos longitudinais ou de teste-reteste a fim de verificar a estabilidade das medidas de interesses profissionais (Lent, Tracey, Brown, Soresi, \& Nota, 2006; Primé \& Tracey, 2010; Rottinghaus, Coon, Gaffey, \& Zytowski, 2007) e explorar a utilidade dos conceitos secundários (Hirschi, 2009, 2010). Hirschi (2010) fez duas aplicações de um instrumento baseado no modelo tipológico de Holland (1997) em uma amostra composta por adolescentes, com intervalo de dez meses. Ao correlacionar os resultados, verificou índices que variaram de 0,46 a 0,77 , sendo o tipo realista o mais estável. De acordo com o autor, os conceitos secundários emergiram como preditores significativos de estabilidade do perfil de interesse, ou seja, a ordem de preferência pelos seis tipos profissionais é significativamente mais estável se o aluno possui interesses que sejam coerentes e nítidos/diferenciados. Além disso, o estudante com interesses mais elevados apresenta pontuação mais estável em cada tipo profissional.

Também, no estudo realizado por Lent et al. (2006), os interesses foram analisados por meio de um instrumento baseado no modelo de Holland (1997). Os autores verificaram a estabilidade dos interesses profissionais em amostras compostas por estudantes italianos do ensino fundamental e médio. O intervalo de aplicação foi de um ano e os coeficientes de estabilidade foram, em geral moderados, com magnitudes entre 0,51 a 0,67 para os alunos do ensino fundamental e 0,60 a 0,84 para o grupo do ensino médio.

Tracey e Robbins (2005) realizaram um estudo sobre a estabilidade temporal e nitidez com uma amostra de estudantes americanos, aplicando o instrumento quando eles estavam no oitavo, no décimo e no décimo segundo ano. Entre o oitavo e décimo ano, os coeficientes de estabilidade variaram de 0,49 a 0,66 e entre o décimo e décimo segundo foi de 0,58 a 0,79 . A variação das médias dos escores ao longo das três medidas foi significativa apenas para uma dimensão de interesse. Quanto à nitidez do perfil, encontraram um aumento da primeira para a segunda medida e, posteriormente, um decréscimo na terceira avaliação. Os autores sugerem a realização de novos estudos, uma vez que o esperado era o aumento contínuo do nível de nitidez.

A partir da revisão de literatura efetuada, foi possível constatar a relevância dos estudos longitudinais em relação a medidas de interesses profissionais, inclusive para a investigação de seus coeficientes de estabilidade e variáveis que podem influenciá-los. Verificou-se também a escassez de estudos brasileiros com foco ou metodologia semelhante. Portanto, o objetivo do presente trabalho é analisar a estabilidade temporal dos escores de interesses medidos pela EAP, bem como dos escores de nitidez/diferenciação e elevação dos interesses, por meio das análises de estabilidade teste-reteste e de nível (Low $\&$ Rounds, 2007).

De acordo com o observado em outras pesquisas, esperam-se coeficientes de correlação moderados a altos entre as medidas (Hirschi, 2010; Lent et al., 2006; Rottinghaus et al., 2007, Sodano, 2015), pouca variação nos escores médios de cada dimensão (Tracey \& Robbins, 2005), e que a associação seja mais acentuada entre o segundo e o terceiro ano (Holland, 1997; Lent et al., 1994; Low et al., 2005; Sodano, 2015). Para os níveis de nitidez e elevação, a expectativa é que sejam crescentes, uma vez que estão associados à estabilidade (Hirschi, 2010; Noronha \& Ambiel, 2015) que tende a aumentar (Holland, 1997; Lent et al., 1994; Low et al., 2005; Sodano, 2015).

\section{Método}

\section{Participantes}

Foram utilizados os dados de 96 estudantes de uma escola pública, colégio de aplicação vinculado a uma universidade pública do estado de Minas Gerais, Brasil. Trata-se de uma amostra selecionada por conveniência. Uma parte dos estudantes frequentou o ensino médio entre os anos de 2008 e 2010 ( $n=54,56,3 \%)$ e outra parte iniciou essa etapa escolar em 2009 e concluiu em 2011 $(n=42,43,7 \%)$. Na amostra geral, verificou-se que 47,9\% $(n=46)$ dos participantes são do sexo masculino e 52,1\% $(n=50)$, do sexo feminino. A média de idade na primeira aplicação foi 15,27 $(D P=0,75)$ anos e, na última, foi de $17,34(D P=0,64)$ anos.

\section{Instrumentos}

Escala de Aconselhamento Profissional EAP (Noronha et al., 2007). A escala contém 61 itens na forma de atividades profissionais, sendo que o participante deve assinalar de 1 (nunca) a 5 (frequentemente) a frequência com que gostaria de desenvolvê-las. Os itens estão divididos em fatores que representam sete dimensões profissionais: (1) Ciências Exatas; (2) Artes/Comunicação; (3) Ciências Biológicas/da Saúde; (4) Ciências Agrárias/Ambientais; (5) Atividade Burocrática; (6) Ciências Humanas e Sociais Aplicadas; e (7) Entretenimento. Uma breve descrição dessas dimensões pode ser encontrada em Noronha et al. (2007) e Lamas e Noronha (2013).

O manual de aplicação da EAP apresenta evidências de validade baseadas na estrutura interna, por meio da 
análise fatorial, em que os itens apresentaram índices de saturação superiores a 0,30 e as sete dimensões explicaram 57,3\% da variância. Evidências baseadas na relação com outras variáveis foram obtidas, de modo concorrente, ao comparar a média nas dimensões com uma variável critério, a saber, carreira universitária frequentada. Quanto à precisão, a consistência interna da escala gerou alfas de Cronbach que variaram de 0,79 a 0,94.

\section{Procedimento}

O projeto de pesquisa foi aprovado pelo Comitê de Ética em Pesquisa - CEP (CAAE: 0265.0.180.000-08). A coleta ocorreu em sala de aula, de forma coletiva, após a explicação dos objetivos da pesquisa, nenhum estudante se recusou a participar. $O$ tempo de aplicação foi de aproximadamente 50 minutos, pois, além da EAP, eles responderam a um questionário com questões sociodemográficas e relacionadas à escolha profissional. As aplicações aconteceram duas vezes ao ano, no primeiro e no segundo semestre, ao longo dos três anos do ensino médio.

Neste trabalho, foi priorizada a coleta do segundo semestre, uma vez que os alunos já tinham cursado um semestre do ano letivo, o que poderia favorecer maior esclarecimento para responder aos instrumentos. Entretanto, algumas aplicações ocorreram com poucos alunos porque a autorização para a coleta foi concedida no final do período letivo, quando a frequência dos estudantes é muito baixa. No caso em que não foi possível utilizar a medida do segundo semestre, utilizou-se aquela coletada no início do ano ( $1^{\circ}$ ano do ensino médio: 5 participantes do primeiro semestre, $5,2 \%$; $2^{\circ}$ ano do ensino médio: 19 estudantes do primeiro semestre, 19,8\%; $3^{\circ}$ ano do ensino médio: 31 alunos do primeiro semestre, $32,3 \%$ ). Assim, o tempo médio em meses entre a primeira (Tempo 1 - T1) e segunda medida (Tempo $2-\mathrm{T} 2$ ) variou de 4 a 20, com média igual a 11,02 $(D P=3,40)$ meses e moda equivalente a 12 , enquanto a média de intervalo entre a segunda (Tempo 2- T2) e a terceira aplicação (Tempo 3- T3) foi de 11,46 $(D P=3,74)$ meses, com variação entre 6 e 20 e moda igual 12.

\section{Análise de dados}

Além de estatística descritiva para fins de apresentação dos resultados do instrumento, foram empregadas provas inferenciais, sendo adotado nível de significância de 0,05. Nesse caso, ao empregar o método de teste-reteste, utilizou-se a correlação de Pearson, sendo que a classificação das magnitudes foi feita a partir de Dancey e Reidy (2006), sendo classificada com força moderada o intervalo entre 0,40 e 0,69 , acima e abaixo desses valores tem-se fraco e forte. Para verificar a variabilidade dos interesses profissionais ao longo do ensino médio, bem como de seus aspectos de nitidez/ diferenciação e elevação foi empregada a ANOVA de medidas repetidas.

Para calcular o nível de interesse em cada dimensão da EAP, foi utilizada a média dos itens correspondentes, portanto, com variação entre um e cinco. $O$ índice de nitidez foi calculado por meio da subtração da média da dimensão de maior escore pela de menor pontuação. $\mathrm{Ou}$ seja, dentre as dimensões profissionais avaliadas, verifica-se o quanto a mais preferida distancia-se da menos preferida (Holland, 1997). Quanto maior o valor, maior o nível de nitidez. Outra forma de obter esse índice é a partir do desvio padrão de todas as dimensões (Hirschi, 2009). Ao verificar que a correlação entre os dois métodos foi de 0,975 , optou-se por utilizar nas análises deste estudo a maneira tradicional, sugerida por Holland (1997). Assim a variação é de 0 a 4.

Elevação refere-se ao nível geral de interesse (Bullock \& Reardon, 2005), assim, ele é obtido por meio da soma dos escores resultantes de cada dimensão. Nesse caso, somaram-se as médias das sete dimensões da EAP, portanto, os índices de elevação poderiam variar entre 7 e 35 .

\section{Resultados}

Inicialmente, apresenta-se a análise descritiva dos escores fatoriais da EAP e dos índices de nitidez e elevação. A Tabela 1 mostra a média e o desvio padrão das três medidas coletadas ao longo do ensino médio.

Tabela 1

Estatística Descritiva dos Escores da EAP

\begin{tabular}{lcccccc}
\hline \multirow{2}{*}{ Variáveis } & \multicolumn{2}{c}{ T1 } & \multicolumn{2}{c}{ T2 } & \multicolumn{2}{c}{ T3 } \\
\cline { 2 - 6 } & Média & DP & Média & DP & Média & DP \\
\hline Ciências Exatas & 2,36 & 0,96 & 2,43 & 1,08 & 2,39 & 1,01 \\
Artes/Comunicação & 2,44 & 0,99 & 2,57 & 1,03 & 2,42 & 1,01 \\
Ciências Biológicas /da Saúde & 2,43 & 1,09 & 2,69 & 1,18 & 2,67 & 1,15 \\
Ciências Agrárias/Ambientais & 2,58 & 0,95 & 2,67 & 0,94 & 2,70 & 0,87 \\
Atividades Burocráticas & 2,57 & 0,80 & 2,67 & 0,83 & 2,70 & 0,91 \\
Ciências Humanas e Sociais & 2,47 & 0,90 & 2,62 & 0,88 & 2,56 & 0,90 \\
Entretenimento & 2,35 & 0,91 & 2,56 & 1,04 & 2,46 & 1,05 \\
Nitidez/Diferenciação & 1,82 & 0,76 & 1,95 & 0,81 & 2,03 & 0,79 \\
Elevação & 17,20 & 4,65 & 18,21 & 4,96 & 17,90 & 4,68 \\
\hline
\end{tabular}

Nota. T1=aplicação no primeiro ano, T2=aplicação no segundo ano, T3=aplicação no terceiro ano 
Os resultados mostram que a média em cada dimensão esteve próxima ao ponto médio e os desvios padrão ao redor de um. É interessante notar que as dimensões Ciências Agrárias/Ambientais e Atividades Burocráticas sempre estiveram entre as maiores pontuações, o que demonstra o maior interesse dos participantes por essas áreas. Ciências Biológicas/da Saúde se junta a essas duas dimensões em T2 e T3.

Os níveis de nitidez foram crescentes, pois a maior média (medida de tendência central do grupo) foi em T3 e a mais baixa em T1, o desvio padrão ficou abaixo de um. Quanto aos índices de elevação dos interesses, verificou-se flutuação dos escores, de forma que, no primeiro ano (T1), o nível foi menor; no segundo (T2), as médias se elevaram e, já no terceiro ano (T3), os valores foram mais baixos que no segundo, porém ainda mais elevados que no primeiro ano do ensino médio. A seguir são apresentadas as análises de estabilidade temporal.

As correlações entre as medidas indicam o nível de estabilidade temporal dos escores provenientes da EAP. A Tabela 2 mostra a correlação entre as medidas obtidas nos três tempos. É importante relembrar que o intervalo médio entre elas foi de 11,02 meses (T1 e T2) e 11,46 meses (T2 e T3).

Ao considerar os coeficientes de estabilidade $\left(r_{T 1 T 2}\right.$ e $r_{\text {T2T3 }}$ ) para as dimensões correspondentes, verificou-se que todas as correlações foram positivas e significativas, de magnitude moderada. Ainda, convém destacar que outras 26 correlações significativas entre dimensões diferentes apresentaram índices fracos e, apenas, quatro coeficientes também moderados.

Tabela 2

Correlação entre as Dimensões ao Longo do Ensino Médio

\begin{tabular}{|c|c|c|c|c|c|c|c|}
\hline EAP & $\begin{array}{c}\text { T2 } \\
\text { Dimensão } 1\end{array}$ & $\begin{array}{c}\text { T2 } \\
\text { Dimensão } 2\end{array}$ & $\begin{array}{c}\text { T2 } \\
\text { Dimensão } 3\end{array}$ & $\begin{array}{c}\text { T2 } \\
\text { Dimensão } 4\end{array}$ & $\begin{array}{c}\text { T2 } \\
\text { Dimensão } 5\end{array}$ & $\begin{array}{c}\text { T2 } \\
\text { Dimensão } 6\end{array}$ & $\begin{array}{c}\text { T2 } \\
\text { Dimensão } 7\end{array}$ \\
\hline $\begin{array}{c}\mathrm{T} 1 \\
\text { Dimensão } 1\end{array}$ & $0,464^{* *}$ & 0,101 & 0,075 & 0,190 & $0,294^{* *}$ & 0,078 & 0,101 \\
\hline $\begin{array}{c}\mathrm{T} 1 \\
\text { Dimensão } 2\end{array}$ & $-0,042$ & $0,415^{* *}$ & 0,105 & 0,161 & 0,033 & $0,291^{* *}$ & $0,356^{* *}$ \\
\hline $\begin{array}{c}\text { T1 } \\
\text { Dimensão } 3\end{array}$ & $-0,041$ & 0,181 & $0,498^{* *}$ & $0,422^{* *}$ & $-0,031$ & 0,158 & 0,167 \\
\hline $\begin{array}{c}\text { T1 } \\
\text { Dimensão } 4\end{array}$ & 0,021 & 0,186 & $0,252^{*}$ & $0,456^{* *}$ & $-0,006$ & 0,171 & $0,214^{*}$ \\
\hline $\begin{array}{c}\text { T1 } \\
\text { Dimensão } 5\end{array}$ & $0,276^{* *}$ & 0,082 & $-0,015$ & 0,142 & $0,435^{* *}$ & 0,168 & $0,239^{*}$ \\
\hline $\begin{array}{c}\mathrm{T} 1 \\
\text { Dimensão } 6\end{array}$ & $-0,168$ & $0,217^{*}$ & 0,049 & 0,166 & 0,054 & $0,423^{* *}$ & $0,272^{* *}$ \\
\hline $\begin{array}{c}\mathrm{T} 1 \\
\text { Dimensão } 7\end{array}$ & 0,118 & $0,288^{* *}$ & 0,104 & $0,209^{*}$ & $0,260^{*}$ & $0,343^{* *}$ & $0,469^{* *}$ \\
\hline $\begin{array}{c}\text { T3 } \\
\text { Dimensão } 1\end{array}$ & $0,434^{* *}$ & $-0,108$ & $-0,020$ & 0,082 & $0,308^{* *}$ & 0,020 & 0,012 \\
\hline $\begin{array}{c}\text { T3 } \\
\text { Dimensão } 2\end{array}$ & $-0,078$ & $0,500^{* *}$ & 0,024 & 0,100 & 0,079 & $0,348^{* *}$ & $0,337^{* *}$ \\
\hline $\begin{array}{c}\text { T3 } \\
\text { Dimensão } 3\end{array}$ & 0,014 & 0,135 & $0,571^{* *}$ & $0,445^{* *}$ & $-0,033$ & 0,121 & 0,132 \\
\hline $\begin{array}{c}\text { T3 } \\
\text { Dimensão } 4\end{array}$ & 0,121 & 0,137 & $0,355^{* *}$ & $0,546^{* *}$ & 0,106 & 0,163 & 0,158 \\
\hline $\begin{array}{c}\text { T3 } \\
\text { Dimensão } 5\end{array}$ & $0,382^{* *}$ & 0,106 & $-0,059$ & 0,103 & $0,503^{* *}$ & $0,254^{*}$ & $0,253^{*}$ \\
\hline $\begin{array}{c}\text { T3 } \\
\text { Dimensão } 6\end{array}$ & 0,026 & $0,363^{* *}$ & $-0,005$ & $0,230^{*}$ & $0,260^{*}$ & $0,497^{* *}$ & $0,311^{* *}$ \\
\hline $\begin{array}{c}\text { T3 } \\
\text { Dimensão } 7\end{array}$ & 0,080 & $0,405^{* *}$ & 0,097 & $0,232^{*}$ & $0,306^{* *}$ & $0,435^{* *}$ & $0,512^{* *}$ \\
\hline
\end{tabular}

Nota. T1=aplicação no primeiro ano, T2=aplicação no segundo ano, T3=aplicação no terceiro ano. 1=Ciências Exatas; 2=Artes / Comunicação; 3=Ciências Biológicas/da Saúde; 4=Ciências Agrárias/Ambientais; 5=Atividade Burocrática; 6=Ciências Humanas/ Sociais Aplicadas; $7=$ Entretenimento. ${ }^{*} p<0,05 ;{ }^{* *} p<0,01$

Destaca-se que, exceto para a dimensão Ciências Exatas, todos os valores absolutos de $r$ gerados pelas associações de T2 e T3 foram mais altos que aqueles originados por T1 e T2. Contudo, ao aplicar o teste de diferença de correlações, a fórmula de Fischer de transformação de $r$ para $z$, esse resultado não foi estatisticamente significativo. 
A fim de analisar estabilidade de nível, ou seja, a extensão em que os escores médios sofreram alteração ao longo dos anos, empregou-se a ANOVA de medidas repetidas. Ao utilizar esse método estatístico, pretende-se, por meio do modelo linear geral, analisar se o perfil encontrado para as médias obtidas é distinto para cada medida aplicada, além de verificar a variabilidade de cada dimensão da EAP. Os resultados são apresentados na Tabela 3.

Tabela 3

ANOVA de Medidas Repetidas pelo Coeficiente de Greenhouse-Geiser

\begin{tabular}{lcccccc}
\hline \multicolumn{1}{c}{ Dimensões da EAP } & SQ & Gl & MQ & $F$ & $p$ & $E^{2} a^{2}$ \\
\hline Ciências Exatas & 0,213 & 2 & 0,108 & 0,168 & 0,841 & 0,002 \\
Artes/Comunicação & 1,346 & 2 & 0,680 & 1,246 & 0,290 & 0,013 \\
Ciências Biológicas/Saúde & 3,901 & 2 & 1,966 & 3,066 & $0,049^{*}$ & 0,031 \\
Ciências Agrárias/Ambientais & 0,760 & 2 & 0,393 & 0,823 & 0,437 & 0,009 \\
Atividades Burocráticas & 0,923 & 2 & 0,468 & 1,145 & 0,320 & 0,012 \\
Ciências Humanas e Sociais & 1,038 & 2 & 0,522 & 1,219 & 0,298 & 0,013 \\
Entretenimento & 2,158 & 2 & 1,091 & 1,964 & 0,144 & 0,020 \\
EAP*Medidas & 2,951 & 7 & 0,422 & 0,424 & 0,888 & 0,003 \\
\hline
\end{tabular}

Nota. $\mathrm{SQ}=$ soma dos quadrados; $\mathrm{gl}=$ graus de liberdade; $\mathrm{MQ}=$ média dos quadrados; Eta $^{2}=$ efeito. ${ }^{*} p<0,05$

Apenas para a dimensão Ciências Biológicas/da Saúde a variação foi significativa, indicando um possível aumento do interesse por essa área quando comparada à média obtida em T1 com aquelas de T2 e T3. Para as demais dimensões, não ocorreram diferenças significativas entre as três medidas e o índice global de efeito não foi expressivo, ou seja, a variabilidade dos escores foi pequena ao longo do tempo. Quanto aos perfis da EAP obtidos em cada aplicação, não houve, também, significância estatística ou efeito a ser considerado (menos de 1\% da variação), ou seja, a distribuição das pontuações nas sete dimensões foi semelhante entre as medidas (Figura 1).

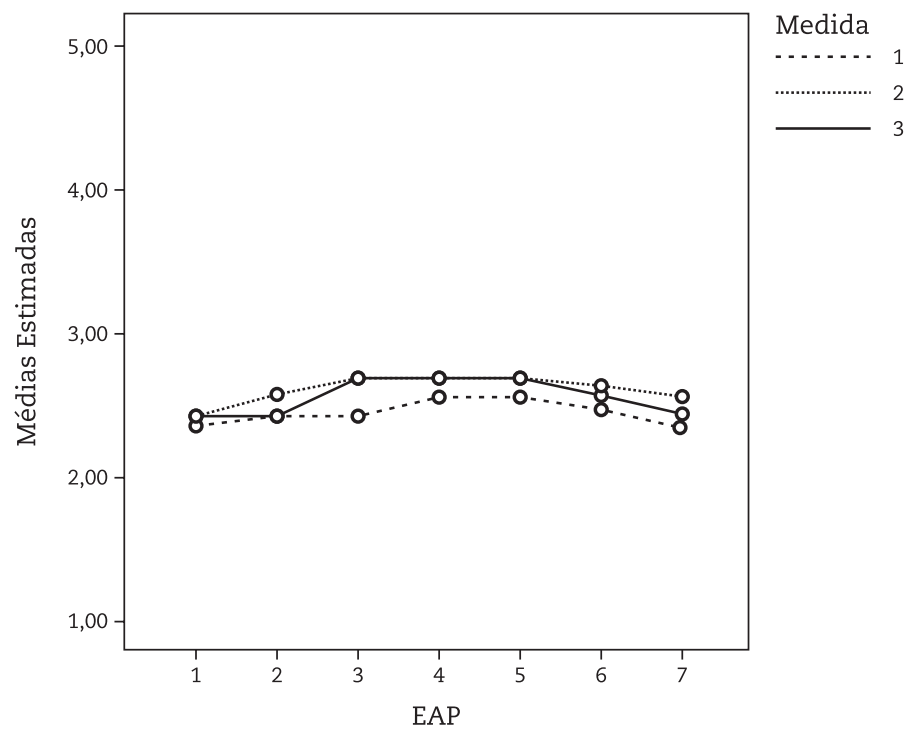

Figura 1. Perfis de interesses gerados pela EAP nos três tempos/medidas

Nota. 1=Ciências Exatas; 2=Artes/Comunicação; 3=Ciências Biológicas/da Saúde; 4=Ciências Agrárias/Ambientais; 5=Atividade Burocrática; 6=Ciências Humanas/Sociais Aplicadas; 7=Entretenimento

A Figura 1 ilustra o perfil gerado para o grupo em cada aplicação da EAP. A estabilidade de nível pode ser visualizada pela proximidade das médias e ao constatar, por exemplo, que, embora na medida 1 (T1) as médias 
tenham sido relativamente mais baixas que nas medidas 2 (T2) e 3 (T3), em todas as mensurações, Ciências Exatas e Entretenimento estiveram entre as dimensões de escores mais baixos. Tais resultados indicam a estabilidade de nível por meio do perfil de interesses gerados pela EAP.

Quanto aos conceitos secundários, verificaram-se correlações positivas e significativas entre as medidas. Para nitidez os índices foram moderados $\left(r_{T 1 T 2}=0,686\right.$; $\left.r_{T 2 T 3}=0,598\right)$ e para elevação encontraram-se magnitudes fracas $\left(r_{T 1 T 2}=0,381 ; r_{T 2 T 3}=0,372\right)$.

A flutuação dos escores de nitidez e de elevação ao longo dos anos foi, também, investigada pela ANOVA de medidas repetidas. De acordo com a análise $(S Q=2,243$; $\left.g l=2 ; \quad M Q=1,215 ; \quad E t a^{2}=0,044\right)$, houve variância significativa para a nitidez $(F[2,175]=4,343, p=0,017)$, ou seja, o nível de diferenciação dos interesses aferido pela EAP mudou ao longo dos anos. Contudo, o tamanho do efeito foi pequeno, indicando que apenas $4 \%$ da variação nos níveis de nitidez podem ser creditados aos diferentes momentos de aplicação.

O teste post hoc de Bonferroni mostra diferença significativa entre T1 e T3 (Tabela 4). Esse resultado indica que o aumento do nível de nitidez do primeiro para o terceiro ano do ensino médio foi significativo e a EAP foi capaz de detectar essa mudança. A ausência de significância com a segunda aplicação (T2) é compreensível ao considerar a posição intermediária que ela ocupa e a estabilidade inerente ao construto referente aos interesses profissionais.

Tabela 4

Teste Post Hoc das Medidas de Nitidez

\begin{tabular}{|c|c|c|c|c|c|c|}
\hline \multirow{2}{*}{ (I) nitidez } & \multirow{2}{*}{ (J) nitidez } & \multirow{2}{*}{$\begin{array}{l}\text { Diferenças das } \\
\text { médias (I-J) }\end{array}$} & \multirow{2}{*}{ Erro Padrão } & \multirow{2}{*}{$p$} & \multicolumn{2}{|c|}{$\begin{array}{l}\text { Limites do intervalo } \\
\text { de confiança de } 95 \%\end{array}$} \\
\hline & & & & & Inferior & Superior \\
\hline \multirow{2}{*}{ T1 } & $\mathrm{T} 2$ & $-0,133$ & 0,064 & 0,119 & $-0,288$ & 0,022 \\
\hline & T3 & $-0,214^{*}$ & 0,082 & 0,032 & $-0,414$ & $-0,014$ \\
\hline \multirow{2}{*}{$\mathrm{T} 2$} & $\mathrm{~T} 1$ & 0,133 & 0,064 & 0,119 & $-0,022$ & 0,288 \\
\hline & T3 & $-0,082$ & 0,073 & 0,802 & $-0,260$ & 0,097 \\
\hline
\end{tabular}

Nota. ${ }^{*} p<0,05$

Quanto à variação dos escores de elevação $\left(S Q=51,714 ; g l=2 ; M Q=25,904 ; E t a^{2}=0,019\right)$, constatou-se que não houve diferença significativa entre as medidas $(F[2,190]=1,877, p=0,156)$. Embora a análise descritiva tenha mostrado que T2 teve maior média que T1 e T3, não é possível afirmar que o nível geral de interesse dos alunos tenha sofrido alteração significativa.

\section{Discussão}

O objetivo deste trabalho foi analisar os resultados de um inventário no que se refere aos níveis de estabilidade dos interesses profissionais, bem como seus escores de nitidez e elevação, ao longo do ensino médio, por meio de um estudo longitudinal. A partir dos resultados, verificou-se que parte das hipóteses foi satisfeita. Inicialmente, é preciso ressaltar que, diferentemente de outros estudos que buscaram investigar a estabilidade temporal de medidas de interesse (Hirschi, 2010; Mansão \& Yoshida, 2006; Primé \& Tracey, 2010; Rottinghaus et al., 2007), foram efetuadas três aplicações do instrumento, pois, tratava-se de um projeto de acompanhamento do desenvolvimento vocacional e não de um estudo transversal.

Ao analisar os coeficientes de estabilidade gerados por meio das associações entre as medidas (T1 vs. T2 e T2 vs. T3), verificaram-se magnitudes moderadas. Esse resultado concorda parcialmente com outros estudos que tiveram um intervalo aproximado (Hirschi, 2010; Lent et al., 2006; Tracey \& Robbins, 2005) ou maior (Rottinghaus et al., 2007) que aquele utilizado na presente pesquisa, pois as correlações encontradas nesses estudos variaram de moderadas a fortes, sendo os valores absolutos mais altos em torno de 0,70. Em um estudo de metanálise, Low et al. (2005) encontraram coeficientes de estabilidade na casa de 0,50 para amostra de adolescentes com idades entre 12 e 17 anos. Mansão e Yoshida (2006) e Primé e Tracey (2010) encontraram intensidade forte a partir de seus instrumentos, porém o período de reaplicação foi curto, apenas uma e duas semanas, respectivamente.

Ressalta-se que a consistência dos escores da EAP foi confirmada em outros estudos (Noronha et al., 2007; Noronha \& Nunes, 2012). Portanto, a compreensão dos níveis moderados de estabilidade aqui encontrados pode ser obtida facilmente recorrendo à literatura. Segundo Urbina (2007), ao realizar um estudo de estabilidade temporal, é necessário considerar o intervalo de aplicação do instrumento e as alterações contextuais e pessoais atreladas a isso, bem como a teoria que fundamenta o construto. Nesse caso, autores como Holland (1997) e Savickas (1999) consideram os interesses profissionais como um traço, e outros destacam o fator desenvolvimental deste, que se torna mais estável, para a maior parte das pessoas, somente ao final da adolescência ou início da idade 
adulta (Lent et al., 1994; Sodano, 2015). Considerando que a amostra deste estudo foi composta por estudantes de ensino médio regular, adolescentes, espera-se que os interesses profissionais desse grupo estejam em desenvolvimento. Dessa forma, as correlações moderadas refletem que, para parte dos estudantes, os interesses estão se estabilizando, mas ainda não se consolidaram.

Ao lado disso, sabe-se que a EAP avalia os interesses em caráter de estado de consciência (Savickas, 1999), isto é, por meio da prontidão para responder a determinados estímulos, especificamente, preferência por determinadas atividades profissionais. Uma vez que os interesses ainda não se estabilizaram completamente, essas preferências vão se modificando, atenuando o caráter de traço refletido pelas medidas ao longo do tempo. Ademais, como afirmam Rounds e Su (2014), considerar os interesses como relativamente estáveis, não quer dizer que eles nunca se modifiquem, significa que são consistentes a um certo grau.

Os valores absolutos dos índices de correlação indicam que a associação das medidas tende a ser maior entre as obtidas no segundo e no terceiro ano (T2 e T3). Entretanto as diferenças não foram estatisticamente significativas e, portanto, não se pode afirmar que houve um aumento significativo da estabilidade dos escores de interesses como visto no estudo de Tracey e Robbins (2005).

Por meio da ANOVA de medidas repetidas, foi possível verificar a baixa variabilidade dos escores nas dimensões ao longo dos três anos, tanto intradimensão quanto entre as dimensões. Apenas para Ciências Biológicas/da Saúde houve aumento significativo do interesse, entre T1 e T2, o que pode estar relacionado à desejabilidade social, já que essa dimensão se refere a atividades da área de saúde, como a medicina. Ao mesmo tempo, a baixa variabilidade mostra que não há grandes oscilações nos escores obtidos por meio desse instrumento, favorecendo a evidência de estabilidade de nível (Low \& Rounds, 2007). Pouca variação nos escores de dimensões de interesses foi, também, encontrada por Tracey e Robbins (2005).

Com relação aos conceitos secundários, as análises de correlação mostraram estabilidade moderada para os índices de nitidez, o que é justificável, pois é um escore para o qual se esperava alguma alteração ao longo tempo. Esse resultado foi a favor das hipóteses elaboradas. O aumento da nitidez dos interesses foi verificado pela análise de variância, o que contribui como uma evidência de fidedignidade desse índice quando aplicado aos escores da EAP. Resultado semelhante foi encontrado por Noronha e Ambiel (2015), que constataram aumento dos níveis de nitidez entre as idades de 15 e 17 anos, a qual corresponde à faixa etária investigada no presente estudo. Entretanto, Tracey e Robbins (2005) encontraram no décimo segundo ano do ensino médio um nível de nitidez menor que no décimo ano, concluindo que esse é um período de reexame da escolha profissional e, por isso, a clareza dos interesses estaria menor.
Para os índices de elevação, verificou-se correlação fraca entre as medidas. E a análise de variância confirma a ausência de significância da diferença entre as medidas, entretanto a expectativa era o aumento da elevação dos interesses com o decorrer do tempo. Esse resultado sugere que, para essa amostra, não houve um padrão de alteração do escore geral de interesse durante o ensino médio. Como sugere Hirschi (2009), mais estudos são necessários acerca da relação com a estabilidade, sendo mais coerente utilizá-lo em associação com traços de personalidade e com a flexibilidade dos interesses (Tracey, 2012).

Em síntese, a maior parte das hipóteses foi satisfeita. Os coeficientes de estabilidade temporal das sete dimensões da EAP foram moderados. Esse resultado é reflexo das bases teóricas utilizadas na construção do instrumento (Savickas, 1999) e do intervalo de tempo adotado (Urbina, 2007). Além disso, como apresentado anteriormente, o resultado foi semelhante ao de outras investigações sobre o tema. Uma vez que os resultados de estabilidade estão em acordo com asserções teóricas e estudos empíricos semelhantes, pode-se concluir também que o instrumento utilizado possui evidências de fidedignidade, sendo capaz de refletir adequadamente a estabilidade dos interesses profissionais. Tal conclusão também se aplica aos escores de nitidez, mas não aos de elevação, para o qual as hipóteses não foram confirmadas. Ressalta-se que este é o primeiro estudo realizado com a EAP que investigou seu escore geral.

Outras pesquisas ainda podem ser realizadas. Seria importante verificar a variação dos escores referentes aos pressupostos secundários em processos de orientação profissional. Relevante, também, uma investigação longitudinal desses conceitos e dos escores da EAP que envolvesse a transição entre os níveis de ensino, médio e superior ou escola-trabalho.

É necessário destacar que o presente estudo possui algumas limitações, por exemplo, foi realizado em apenas uma escola, com pequeno número de alunos. Ainda, em relação ao momento de aplicação da medida utilizada nesta pesquisa, uma pequena porcentagem de participantes não teve a avaliação do final do ano letivo, sendo utilizada aquela obtida no início do ano, o que causou variação no intervalo entre as aplicações.

Contudo, há que se destacar que são poucos os estudos longitudinais realizados com inventários de interesses profissionais no Brasil, possivelmente, pela dificuldade da implantação desse tipo de delineamento (Mota, 2010). Assim, não foram encontrados outros trabalhos nacionais com características similares, instrumento ou intervalo de tempo, para realizar a comparação dos resultados obtidos. Espera-se que este primeiro trabalho possa impulsionar os pesquisadores a empreenderem delineamentos semelhantes, pois, como afirmou Hansen (2005), esse tipo de estudo contribui para a segurança da utilização de inventários de interesses na prática do psicólogo. 


\section{Referências}

Bullock, E. E., \& Reardon, R. C. (2005). Using profile elevation to increase the usefulness of the self-directed search and other inventories. Career Development Quarterly, 54(2), 175-183. doi: 10.1002/j.2161-0045.2005.tb00149.x

Dancey, C. P., \& Reidy, J. (2006). Estatística sem matemática para psicologia usando SPSS para Windows. Porto Alegre: Artmed.

Fuller, B. E., Holland, J. L., \& Johnston, J. A. (1999). The relation of profile elevation in the self-directed search to personality variables. Journal of Career Assessment, 7(2), 111-123. doi: 10.1177/106907279900700202

Hansen, J. C. (2005). Assessment of interests. Em S. D. Brown \& R. W. Lent (Eds.), Career development and counseling: putting theory and research to work (pp. 281-304). Hoboken, NJ: John Wiley \& Sons.

Hirschi, A. (2009). Development and criterion validity of differentiated and elevated vocational interests in adolescence. Journal of Career Assessment, 17(4), 384-401. doi: 10.1177/1069072709334237

Hirschi, A. (2010). Individual predictors of adolescents' vocational interest stabilities. International Journal for Educational and Vocational Guidance, 10(1), 5-19. doi: 10.1007/s10775-009-9171-2

Holland, J. L. (1997). Making vocational choices: A theory of vocational personalities and work environments (3rd ed.). Odessa, FL: Psychological Assessment Resources.

Jaensch, V. K., Hirschi, A., \& Spurk, D. (2016). Relationships of vocational interest congruence, differentiation, and elevation to career preparedness among university students. Zeitschrift für Arbeits und Organisationspsychologie, 60(2), 79-89. doi: 10.1026/0932-4089/a000210

Lamas, K. C. A., \& Noronha, A. P. P. (2013). Relações entre disciplinas escolares e interesses profissionais. Atas do XII Congresso Internacional Galego-Português de Psicopedagogia. Braga: Centro de Investigação em Educação (CIEd)/Instituto de Educação Universidade Minho, 428-440.

Lent, R. W., Brown, S. D., \& Hackett, G. (1994). Toward a unifuing social cognitive theory of career and academic interest, choice and performance. Journal of Vocational Behavior, 45(1), 79-122. doi: 10.1006/jvbe.1994.1027

Lent, R. W., Tracey, T. J. G., Brown, S. D., Soresi, S., \& Nota, L. (2006). Development of interests and competency beliefs in Italian adolescents: An exploration of circumplex structure and bidirectional relationships. Journal of Counseling Psychology, 53(2), 181-191. doi: 10.1037/0022-0167.53.2.181.

Low, K. S. D., \& Rounds, J. (2007). Interest change and continuity from early adolescence to middle adulthood. International Journal of Educational and Vocational Guidance, 7(1), 23-36. doi: 10.1007/s10775-006-9110-4.

Low, K. S. D., Yoon, M., Roberts, B. W., \& Rounds, J. (2005). The stability of vocational interests from early adolescence to middle adulthood: A quantitative review of longitudinal studies. Psychological Bulletin, 131(5), 713-737. doi: 10.1037/0033-2909.131.5.713

Mansão, C. S. M., \& Yoshida, E. M. P. (2006). SDS-Questionário de busca autodirigida: Precisão e validade. Revista Brasileira de Orientação Profissional, 2(1), 67-79. Recuperado de http://www.redalyc.org/html/2030/203016895007/

Mota, M. M. P. E. (2010). Metodologia de pesquisa em desenvolvimento humano: velhas questões revisitadas. Psicologia em Pesquisa, 4(2), 144-149. Recuperado de http://pepsic.bvsalud.org/scielo.php?pid=S1982-12472010000200007\&script=sci_abstract

Noronha, A. P. P., \& Ambiel, R. A. M. (2015). Level of differentiation of vocational interest's profiles: Comparative study by age and schooling in a brazilian sample. Paidéia (Ribeirão Preto), 25(60), 49-56. doi: 10.1590/1982-43272560201507

Noronha, A. P. P., Freitas, F. A., \& Ottati, F. (2003). Análise de instrumentos de avaliação de interesses profissionais. Psicologia: Teoria e Pesquisa, 19(3), 287-291. doi: 10.1590/S0102-37722003000300011

Noronha, A. P. P., \& Nunes, M. F. O. (2012). Escala de Aconselhamento Profissional: Análise com estudantes de ensino médio. Fractal: Revista de Psicologia, 24(2), 405-422. doi: 10.1590/S1984-02922012000200013.

Noronha, A. P. P., Pinto, L. P., \& Luca, L. (2015). Perfis profissionais de universitários da área de biológicas e afins: interação entre curso e interesses. Psicologia desde el Caribe, 32(2), 286-298. Recuperado de http://www.scielo.org.co/pdf/psdc/v32n2/v32n2a07.pdf

Noronha, A. P. P., Ottati, F., Mansão, C. S. M., \& Cezar, E. O. (2011). Aplicação da Escala de Aconselhamento Profissional em Estudantes Universitários. Acta Colombiana de Psicologia, 14(1), 155-164. Recuperado de http://www.scielo.org.co/scielo.php?script=sci_arttext\&p $\mathrm{id}=\mathrm{S} 0123-91552011000100014$

Noronha, A. P. P., Sisto, F. F., \& Santos, A. A. A. (2012). Validación de una escala de aconsejamiento profesional. Persona, 15, $157-172$. Recuperado de http://repositorio.ulima.edu.pe/handle/ulima/2221

Noronha, A. P., Sisto, F., \& Santos, A. A. A. (2007). Escala de Aconselhamento Profissional - EAP. Manual Técnico (Brasil). Itatiba, SP: Vetor Editora.

Primé, D. R., \& Tracey, T. J. G. (2010). Psychometric properties of the career clusters interest survey. Journal of Career Assessment, 18(2),177188. doi: $10.1177 / 1069072709354202$

Rottinghaus, P. J., Coon, K. L., Gaffey, A. R., \& Zytowski, D. G. (2007). Thirty-year stability and predictive validity of vocational interests. Journal of Career Assessment, 15(1), 5-22. doi: 10.1177/1069072706294517.

Rounds, J., \& Su, R. (2014). The nature and power of interests. Current Directions in Psychological Science, 23(2) 98-103. doi: $10.1177 / 0963721414522812$.

Sartori, F. A., Noronha, A. P. P., Godoy, S., \& Ambiel, R. A. M. (2010). Interesses profissionais de jovens de ensino médio: Estudo correlacional entre a escala de aconselhamento profissional e o self-directed search carrier explorer. Estudos de Psicologia, 27(2), 215-225. doi: 10.1590/ S0103-166X2010000200009.

Savickas, M. L. (1999). The Psychology of Interests. Em M. L Savickas \& A. R. Spokane, (Eds.), Vocational interests: meaning, measurement and counseling use (pp.19-56). Palo Alto, CA: Davies-Black.

Sisto, F. F., Santos, A. A. A., \& Noronha, A. P. P. (2009). Escala de aconselhamento profissional: Carreira universitária como evidência de validade de critério. Revista Galego-Portuguesa de Psicoloxía e Educación, 17(1,2), 185-194. Recuperado de https://www.researchgate.net/ publication/277829826_Escala_de_aconselhamento_profissional_carreira_universitaria_como_evidencia_de_validade_de_criterio

Sodano, S. M. (2015). Meaning, measurement, and assessment of vocational interests for career intervention. Em P. J. $\bar{H}$ Hartung, M. L. Savickas \& W. B. Walsh (Eds.), APA Handbook of Career Intervention: Volume 1. Foundations, (pp. 281-301). Washington, DC, US: American Psychological Association

Super, D. E. (1957). The Psychology of Careers. New York: Harper and Row.

Tracey, T. J. G. (2012). Problems with single interest scales: Implications of the general factor. Journal of Vocational Behavior, 81(3), 378-384. doi: $10.1016 /$ j.jvb.2012.10.001. 
Tracey, T. J. G., \& Robbins, S. B. (2005). Stability of interests across ethnicity and gender: A longitudinal examination of grades 8 through 12. Journal of Vocational Behavior, 67(3), 335-364. doi: 10.1016/j.jvb.2004.11.003.

Wang, M., \& Wanberg, C. R. (2017). 100 years of applied psychology research on individual careers: From career management to retirement. Journal of Applied Psychology, 102(3), 546-563. doi: 10.1037/apl0000143.

Urbina, S. (2007). Fundamentos da testagem psicológica. Porto Alegre: Artmed.

\section{Sobre as autoras}

Karen Cristina Alves Lamas é psicóloga, doutora em Psicologia pela Universidade São Francisco. Atualmente é professora do curso de graduação em psicologia da Universidade Salgado de Oliveira, Juiz de Fora.

Ana Paula Porto Noronha é psicóloga, doutorado em Psicologia Ciência e Profissão pela Pontifícia Universidade Católica de Campinas. Atualmente é Professora Associada do Programa de Pós-graduação Stricto Sensu em Psicologia da Universidade São Francisco. 